\title{
Relationships between social withdrawal and facial emotion recognition in neuropsychiatric disorders
}

Alejandro Torre-Luque ( $\boldsymbol{D}$ af.delatorre@ucm.es )

Universidad Complutense de Madrid

Alba Viera-Campos

Hospital Universitario de La Princesa

Amy C. Bilderbeck

p1 Vital Ltd.

Maria Teresa Carreras

Hospital Universitario de La Princesa

Jose Vivancos

Hospital Universitario de La Princesa

Covadonga M. Diaz-Caneja

Hospital General Universitario Gregorio Marañón

Moji Aghajani

Amsterdam UMC

Ilja M. J. Saris

Amsterdam UMC

Andreea Raslescu

p1 Vital Ltd.

Asad Malik

p1 Vital Ltd.

Jenna Clark

University of Nottingham

Brenda W. J. H. Penninx

Amsterdam UMC

Nic Van der Wee

Leiden University Medical Center

Inge Winter-van Rossum

King's College London

Bernd Sommer

Boehringer Ingelheim (Germany)

Hugh Marston

Eli Lilly (United Kingdom)

Gerard R. Dawson 
p1 Vital Ltd.

\section{Martien J. Kas}

University of Groningen

Jose Luis Ayuso-Mateos

Centro de Investigación Biomédica en Red de Salud Mental

\section{Celso Arango}

Hospital General Universitario Gregorio Marañón

\section{Research Article}

Keywords: Neuropsychiatric disorder, social functioning, schizophrenia, Alzheimer's disease, emotion recognition, social cognition, PRISM study

Posted Date: February 22nd, 2021

DOl: https://doi.org/10.21203/rs.3.rs-209509/v1

License: (c) (i) This work is licensed under a Creative Commons Attribution 4.0 International License. Read Full License

Version of Record: A version of this preprint was published at Progress in Neuro-Psychopharmacology and Biological Psychiatry on October 1st, 2021. See the published version at https://doi.org/10.1016/j.pnpbp.2021.110463. 


\section{Abstract}

This study aimed to investigate the role of social withdrawal in emotion recognition of patients with schizophrenia (SZ) or probable Alzheimer's disease (AD). A sample of 156 participants was recruited: SZ patients $(n=53)$, AD patients $(n=46)$, and two age-matched control groups (SZc, $n=29 ; \mathrm{ADc}, n=28)$. All participants provided self-report measures of loneliness and social functioning, and completed a facial emotion detection task. As a result, neuropsychiatric patients (both groups) showed poorer performance in detecting both positive and negative emotions compared with their healthy counterparts $(p<.01)$. Social withdrawal was associated with higher accuracy in negative emotion detection, across all groups. Additionally, neuropsychiatric patients with higher social withdrawal showed lower positive emotion misclassification. Our findings help detail the similarities and differences in social function and facial emotion recognition in two disorders rarely studied in parallel, AD and SZ. Transdiagnostic patterns in these results suggest that social withdrawal is associated with heightened sensitivity to negative emotion expressions, potentially reflecting hypervigilance to social threat. Across the neuropsychiatric groups specifically, this hypervigilance associated with social withdrawal extended to positive emotion expressions, an emotional-cognitive bias that may impact social functioning in people with severe mental illness.

\section{Background}

The impact of social withdrawal and social isolation is quite evident in everyday life. Several authors postulate that social withdrawal factors may alter the way in which individuals interpret the social world. ${ }^{1,2}$ Social withdrawal factors (social disengagement, lack of interpersonal relationships and loneliness) may trigger implicit hypervigilance for social threats (possibly related to unmet needs of belonging) ${ }^{3,4}$ As a result, a pattern of heightened sensitivity to particular social cues (e.g., signs of disapproval, such as several facial expressions) emerges. This way of perceiving the social world makes it more likely for individuals to engage in behavioural repertoires that subsequently lead to more negative interactions and social isolation. Vanhalst et al. ${ }^{5}$ showed that perceived social isolation (loneliness) in adolescents was associated with increased sensitivity (reduced intensity required for emotion decoding) to detect negative (sadness and fear) emotional faces, after controlling for relevant covariates (sex and psychopathology). Similarly, Bangee and Qualter ${ }^{6}$ found that loneliness was associated with an earlier orientation to negative faces (angry faces) in crowds, using an eye tracking paradigm. The authors concluded that a loneliness-related cognitive bias may exist. This bias may help individuals avoid potential situations of social threatening quicker.

The Research Domain Criteria (RDoC) initiative aims at stimulating research into common (transdiagnostic) impairments across mental disorders in an attempt to better understand the nature of mental health. ${ }^{7}$ In this regard, some studies have suggested that social dysfunction may be a candidate for transdiagnostic marker of mental illness, as equivalent impairments have been shown at the social domain between several disorders, such as attention-deficit/hyperactivity disorder and autism spectrum 
disorder, or between depression and schizophrenia. ${ }^{8,9}$ The transdiagnostic nature of social withdrawal factors deserves being studied in neuropsychiatric disorders, such as schizophrenia (SZ) and dementia, which are featured by evident deficits in social cognition. First, numerous studies point that social withdrawal may predispose for both dementia (specifically Alzheimer's disease [AD]) and schizophrenia

emergence or symptom aggravation. ${ }^{10-14}$ Second, psychotic symptoms are highly related to feelings of social withdrawal and isolation. ${ }^{15}$ Finally, several studies have shown deficits in decoding facial emotions in schizophrenia patients as well as in Alzheimer's patients. ${ }^{16-19}$ Thus, emotion recognition deficits may lead to social isolation and feelings of loneliness. In turn, social isolation factors may similarly influence the pre-existing emotion recognition deficits, leading to attentional, confirmatory, and memorial biases in these patients.

This study aimed to investigate the transdiagnostic role of social withdrawal (social disengagement, lack of interpersonal relationships and loneliness) in patients with $S Z$ and $A D$, by examining its relationship with emotion recognition. First, we hypothesised that neuropsychiatric patients would show worse performance on emotion recognition tasks than their healthy control counterparts. Additionally, we expected social withdrawal to lead to better performance (i.e., higher accuracy rates) in negative emotion recognition, regardless of the diagnosis. Finally, we speculated that a positive relationship between correct recognition of positive emotions and social withdrawal factors would exist in neuropsychiatric patients. In other words, social withdrawal would lead to similar impairments in both positive and negative emotion recognition in patients with $S Z$ and $A D$.

\section{Results}

Table 1 displays the sociodemographic and clinical features of study participants. Comorbid emotional disorders were present in more SZ cases in comparison to its age control group (SZc). The SZc participants remained more years in formal education and showed higher scores in the DSST, the SFS total score, and the social engagement and the interpersonal behaviour subscales. On the other hand, the SZc particpants showed lower levels of depressive symptoms and loneliness than their counterparts. Finally, the AD participants exhibited lower scores in the general cognition and interpersonal behaviour scales than their matched controls, but higher depressive symptoms.

\section{Performance on emotion recognition task}

Table 2 displays the scores in accuracy and misclassification across the study groups. Group differences were observed for all the FERT outcomes except for the negative emotion misclassification rate (univariate $p>.05$ ). In general terms, participants from the AD showed the lowest accuracy rate for both types of emotions, as well as the highest levels of misclassification of positive emotions. The SZc showed the lowest positive emotion misclassification rate and the highest accuracy rate of negative emotion recognition across groups. The SFS total score did not correlate with performance on FERT task $(p>.17)$. 


\section{Relationship between emotion recognition and social withdrawal}

Multilevel regression models with covariates showed better fit to data than the unconstrained ones for both positive and negative emotion accuracy rates and the positive emotion misclassification rate (see the AIC for all the models in Table 3). This means that covariates are relevant to predict the abovementioned FERT outcomes beyond the effect of a random-effects component (variability due to study group). Regarding the negative emotion misclassification rate, the unconstrained model showed lower AIC (AIC $=1080.80)$ than the model with sociodemographic covariates (AIC $=1083.88)$ and the full (all covariates) model (AIC $=1082.47)$. The model showed a fixed effect of $B=9.15, S E=0.65, t(2.37)=$ $13.98, p<.01$; and random-effects $S D=0.61$. The model with sociodemographic covariates fitted better to data than the other nested models for the positive emotion accuracy rate. On the other hand, the fullcovariate model fitted better for the positive emotion misclassification rate and the negative emotion accuracy rate (see Table 3).

The accuracy rate of recognising positive emotions was predicted by sex (women showing higher accuracy), $B=3.89, p<.01$; and age (the higher the accuracy, the younger the participant), $B=-0.17, p<$ .05. On the other hand, the positive emotion misclassification rate was predicted by $\operatorname{sex}, B=-3.48, p<.01$; and general cognition (higher misclassification with lower cognition scores), $B=-0.25, p<.01$. Additionally, the social engagement covariate showed a positive loading (Table 3 ), $B=0.16, p<.05$. This points to higher rates of misclassification with higher engagement scores. Group-specific linear univariate regression was conducted using the predicted misclassification rate (derived from the multilevel regression model) as a criterion and the social engagement score as a predictor (essentially to estimate group-specific loadings of this covariate). Bonferroni correction was applied to identify significant predictor loadings $(.05 / 4=.0125)$. As a result, Group-specific univariate regression confirmed this effect on predicted misclassification rates for $\mathrm{SZ}(B=0.12, t=4.55, p<.01)$ and $\mathrm{AD}(B=0.20, t=5.35, p<.01)$ participants (see Figure 1).

The accuracy in detecting negative emotions was predicted by $\operatorname{sex}(B=4.73, p<.01)$ and general cognition (higher levels of accuracy with higher levels of cognition), $B=0.44, p<.01$; and social engagement (higher accuracy with lower engagement scores), $B=-0.31, p<.01$. Group-specific (see Figure 1) regression confirmed this effect on predicted accuracy rates for all the study groups, except the $\mathrm{ADc}(\mathrm{SZc} ; B=-0.26, t=-2.83, p<.01 ; \mathrm{SZ}: B=-0.27, t=-5.02, p<.01 ; \mathrm{ADc}: B=-0.14, t=-1.96, p=.061 ; \mathrm{AD}$ : $B=-0.33, t=-5.18, p<.01)$.

\section{Discussion}

This study aimed to find the common (transdiagnostic) pathways in SZ and AD. More concretely, we were interested in clarifying the association between social withdrawal factors and a pivotal process of social cognition: emotion recognition from facial expressions.

Our study revealed that $A D$ showed the worst performance (i.e., lowest accuracy for both types of emotions and highest misclassification rate of positive emotions) in the emotional recognition task. Note 
that the misclassification rate does not account for positive emotions being necessarily misclassified as negative ones but also as neutral. In line with numerous studies, emotion recognition deficits are characteristic of $A D$ patients and may be explained by some alterations in activity of some brain structures, such as increased amygdala activation, and atrophy in fusiform gyrus or dorsal pallidum. ${ }^{36-38}$ On the other hand, the SZ participants showed lower accuracy of negative emotions than their age counterparts, as well as higher misclassification rate of positive emotions. Schizophrenia patients tend to show impaired facial emotion decoding abilities potentially due to diminished activation of amygdala, superior temporal sulcus, fusiform gyrus and hippocampal regions, anterior cingulate cortex and medial prefrontal areas; areas highly involved in emotion decoding. ${ }^{39-41}$

Moreover, our study provided some evidence on the relationship between social withdrawal factors and social cue processing. Results from regression models demonstrated that social withdrawal (understood as social disengagement) had an important role in negative emotion decoding, across all patient and control study groups. Specifically, higher accuracy in detecting concrete negative emotions (i.e., sadness, fear, disgust, anger) was associated with higher social withdrawal across study groups. These results go in line with the hypervigilance to social threat corollaries. ${ }^{1,2,4}$ Our study extends these findings in research on healthy adolescents and adults to neuropsychiatric disorder patients. ${ }^{3,5,6}$ Thus, it seems that the increased sensitivity associated with social withdrawal observed in negative emotion decoding is evident across the study groups, regardless of neuropsychiatric disorder (i.e., SZ or AD) is present.

Interestingly, we provide some additional evidence on positive emotion decoding in neuropsychiatric disorders and the role here of social withdrawal. Increased social withdrawal (or reduced social engagement according to the SFS subscale labelling) was associated with positive emotion detection, in terms of reduced misclassification. This pattern was observed in both SZ and AD patients, but not in healthy controls. These findings point social withdrawal to be a potential transdiagnostic marker of impaired emotion recognition in SZ and AD patients. Some authors postulate that social cognition impairment constitutes a key marker of neuropsychiatric disorders (e.g., SZ, AD, bipolar disorder, autism). ${ }^{42,43}$ According to the hypervigilance to social threat hypothesis, people with high levels of social withdrawal tend to be hypersensitive to social cues in an attempt to avoid needs of belonging being unmet. Social factors (e.g., social isolation and social network size) have been linked with the activation of relevant cortical areas involved in social cognition (particularly in facial emotion decoding and nonverbal cue integration), such as ventromedial frontal gyrus, amygdala, and superior temporal gyrus. ${ }^{41,44-46}$ This points to common neural circuitry between social withdrawal factors and social cognition processes. Alterations in these circuits have been observed across neuropsychiatric disorders. We speculate that $S Z$ and $A D$ participants with high levels of social withdrawal showed a pattern of heightened sensitivity even when decoding positive emotions from facial expressions. This may interact with pre-existing emotion decoding deficits observed in patients with neuropsychiatric disorders. We could discard the influence of either clinical depression (or high levels of symptoms) or social anxiety on our results as such diagnoses were not endorsed after delivering the diagnostic interview. 
Our study provides some evidence on an association of key processes of social cognition, such as emotion recognition in social contexts, and behavioural aspects of social withdrawal key processes of social cognition, such as emotion recognition in social contexts. Emotion recognition from facial expressions was shown to be impaired in SZ and AD. Social withdrawal factors were related to better performance on negative emotion recognition for both health controls and neuropsychiatric patients. This indicates that social withdrawal factors may make confirmation bias (i.e., increased ability for negative emotion detection with the expectation of being socially rejected) emerge in an attempt to prevent potential social rejection. This bias is also observed for positive emotion recognition in individuals with neuropsychiatric disorders. In this regard, emotion recognition deficits in both SZ and AD may be maintained by same (transdiagnostic) pathways in which social withdrawal have a crucial role. Whilst better emotion recognition could have causally produced higher levels of social withdrawal in our sample, this is not supported by several of our observations. First, the effect of social withdrawal factors on emotion recognition was evident even in individuals who showed poor performance in the emotion recognition task (SZ and AD participants). Second, the influence of social withdrawal factors on emotion recognition was shown for negative emotions in all the study groups, even in the healthy control ones; this is congruent with the hypervigilance to social threat hypothesis. However, our results should be replicated using longitudinal design studies to fully discard reverse causation hypotheses.

This study shows some shortcomings. First, some confounding factors were not taken into account, such as impulsivity or decision-making abilities. We discarded the influence of these factors on the relationship between social withdrawal and performance in emotion recognition tasks. This is because these factors may show an overall effect on recognition outcomes across emotions; in other words, the rate of accuracy and misclassification would be equally affected by impulsive responses across all the emotions. Second, our results failed to show significant effects of all the social withdrawal factors across the outcomes. Most studies investigated the role of loneliness (considered as a subjective dimension of social withdrawal) have revealed a significant effect on facial emotion recognition. ${ }^{6,47}$ However, they did not consider any other social withdrawal factors (social withdrawal is considered a more objective dimension). Potential correlations between social withdrawal factors may mask distinctive effects of each of the social withdrawal factors. Finally, further evidence should be included from brain activity measures on face emotion decoding tasks (e.g., fMRI and EEG tasks used in PRISM) to support our results regarding the role of social withdrawal factors.

To sum up, our study stresses how negative social withdrawal and isolation may be critical for neuropsychiatric patients, as they affect basic processes (face emotion decoding) involved in social cognition. Social withdrawal factors may be potential transdiagnostic targets due to their relationship with emotion recognition impairment in both $S Z$ and $A D$. On the other hand, this study provides some evidence in favour to develop interventions promoting social integration of people with neuropsychiatric disorder. In this vein, initiatives or interventions focused on reducing social withdrawal and isolation of psychotic/neurodegenerative spectrum people may contribute to palliate social cognition deficits. This directly leads to improve the quality of life and social adjustment of people with severe mental illness. 


\section{Methods}

\section{Sample}

A sample of 156 participants from the PRISM (Psychiatric Ratings using Intermediate Stratified Markers) Study (http://prism-project.eu) was used. ${ }^{20,21}$ The sample comprised four groups of participants: individuals with schizophrenia (SZ; $n=53,71.70 \%$ men; $m=30.45$ years, $s d=6.06$ ); participants with a probable diagnosis of Alzheimer's disease (AD; $n=46,55.56 \%$ men; $m=68.80$ years, $s d=7.13$ ); and two age-matched control groups (SZc: $n=29,58.62 \%$ men; $m=28.72$ years, $s d=7.40$; ADc: $n=28,53.57 \%$ men; $m=68.80$ years, $s d=7.13)$. All the participants performed a face emotion decoding task. Diagnosis of SZ participants was confirmed according to the criteria of the Diagnostic and Statistical Manual of Mental Disorders (DSM-IV) ${ }^{22}$ by means of the MINI International Neuropsychiatric Interview. ${ }^{23}$ The diagnosis of $A D$ patients was confirmed according to the criteria of $A D$ as outlined by the National Institute on Aging (NIA) and the Alzheimer's Association (AA). ${ }^{24}$ Participants with either a current diagnosis of major depressive disorder or high levels of depressive symptoms (QIDS $\geq 16$ ) were excluded.

The sample was recruited from two hospitals in Spain (Gregorio Maranon University Hospital and La Princesa University Hospital) and three centres in the Netherlands (University Medical Center Utrecht, VU University Medical Center Amsterdam and Leiden University Medical Center). All the study protocols were approved by the Clinical Research Ethics Committee of the research sites (i.e., Gregorio Maranon University Hospital, La Princesa University Hospital, University Medical Center Utrecht, VU University Medical Center Amsterdam and Leiden University Medical Center). Moreover, all the participants (or their legal guards) provided a signed written consent form to participate in this study. informed consent was obtained from all participants and/or their legal guardians. All the study protocols were performed in accordance with the Declaration of Helsinki.

\section{Data collection instruments and tasks}

All the data used in this study were collected in the Assessment visit 1 of the PRISM study. ${ }^{20}$ First, a semistructured interview on sociodemographic and medical data was conducted (i.e., age, sex, race, years of education). Where available, medical notes provided further detail of mental and physical diseases and medication. The MINI International Neuropsychiatric Interview, Screening version (M.I.N.I.-Screen) ${ }^{25}$ was used to explore the presence of psychiatric disorders among study participants. The interview was administered by trained psychiatric researchers.

Three scales were administered to measure social functioning: the social engagement/withdrawal and the interpersonal behaviour subscales ( $a=.76$, for both subscales) of the Social Functioning Scale $(\mathrm{SFS})^{26}$ and the Loneliness and Affiliation Scale (LAS; $\left.\mathrm{a}=.88\right) .{ }^{27}$ Other scales used were: the Quick Inventory of Depressive Symptomatology, Self-reported version (QIDS-SR16) to assess depressive symptoms (Cronbach's $a=.86$ ); ${ }^{28}$ the Positive and Negative Syndrome Scale (PANSS) to measure 
psychotic symptoms in the SZ sample (Cronbach's a between .83 and .87); ${ }^{29}$ and the Mini-Mental State Examination (MMSE) to screen cognitive impairment in the AD sample (Livingston's $r$ between .79 and .80). ${ }^{30,31}$

Two performance tasks were carried out, both delivered on the online P1vital ${ }^{\circledR}$ ePRO system. The Digit Symbol Substitution Task (DSST) was used to measure general cognition. ${ }^{32}$ The task draws on aspects of cognition, including speed of processing and working memory. The task involves individual matching symbols to numbers according to a key. A score is constructed by adding up the number of correct symbols coded within 90 seconds. Afterwards, participants completed the computer-generated task Facial Expression Recognition Task (FERT). ${ }^{20,33,34}$ An individual is asked to indicate whether the depicted face (displayed very briefly, $0.5 \mathrm{~s}$ each) is showing either an emotion of happiness, sadness, fear, disgust, surprise, anger; or no emotional expression (i.e., neutral face). The pictures of faces displayed the emotional expressions at 10 different intensities (10\% to $100 \%$ in steps of $10 \%$ ). Patients were asked to categorise the expression of the faces as one of the emotions listed above. Emotion presentation order was random. Emotion presentation order was random. Two main endpoints can be obtained from the FERT task, for each emotion: accuracy rate (number of the emotion responses when presented divided by the number of times faces with this emotion is presented, expressed as a percentage) and misclassification rate (number of the emotion responses when not presented divided by the number of times other emotions or neutral faces are presented, expressed as a percentage).

\section{Procedure}

Participants were recruited when attending either neurology or psychiatry unit visits at the abovementioned healthcare centres. Participants were asked to complete the screening instruments (sociodemographic interview, QIDS-SR16, MMSE, PANSS) in the Assessment visit 1, upon completion of informed consent forms. Additionally, the psychiatric interview (M.I.N.I.-Screen) was conducted. A second visit was scheduled. This visit involved participants completing the questionnaires on social functioning factors (LAS, SFS) and the performance tasks (DSST and FERT). Further details on all protocols implemented in the PRISM study are displayed elsewhere. ${ }^{20}$

\section{Data analysis}

Two multivariate analyses of covariance (ANCOVA) were conducted to study between-group differences on emotion recognition performance (accuracy rate and misclassification rate). A multivariate ANCOVA was conducted for positive emotion recognition, and another for negative emotion outcomes. The study group (with three levels: SZ, AD, controls) was considered as a between-group factor for both ANCOVAs. Age group (younger participants vs. older) was used as a covariate. Bonferroni corrected $t$ tests were used to ascertain pairwise differences between study groups, considering the four groups ( $S Z, A D, S Z c, A D c)$, setting the multiple-comparion $p$ value, $.05 / 6=.0083$. Effect size estimates were the $\mathrm{h}^{2}$ partial and Cohen's d. 
Multilevel linear regression was used to study the influence of social isolation factors on emotional face decoding (accuracy rate and misclassification rate outcomes). This approach allows for studying the fixed effect of these factors controlling for random effects derived from a grouping (level) factor. Sociodemographic factors (sex and age), general cognition (DSST score), comorbidity with emotional disorders, depressive symptoms (QIDS-SR16 score) and the social isolation scores were used as covariates. The study group was used as a level factor. The recruitment site was used as a weighting factor. A model comparison rationale was followed. The full regression model (sociodemographic, DSST score, comorbidity with emotional disorders, depressive symptoms and social functioning covariates) was compared with an unconstrained model and a model with sociodemographic (sex and age) covariates. The Akaike information criterion (AIC) was used for model comparison (lower AIC values indicating better model fit) and the conditional $R^{2}$ as an effect size estimate. ${ }^{35}$

All the analyses were performed by using the R software x64 3.0.1.

\section{Declarations}

\section{Conflict of interest disclosure}

Dr. Arango has been a consultant to or has received honoraria or grants from Acadia, Angelini, Gedeon Richter, Janssen Cilag, Lundbeck, Minerva, Otsuka, Roche, Sage, Servier, Shire, Schering Plough, Sumitomo Dainippon Pharma, Sunovion and Takeda.

Dr. Gerard Dawson is co-owner and an employee of P1vital Ltd.

The authors declare that they do not have any other potential conflict of interest to disclose.

\section{Funding}

This study received funding from the European Union Seventh Framework Program under grant agreements and European Union H2020 Program under the Innovative Medicines Initiative 2 Joint Undertaking (grant agreement No 115916, Project PRISM, and grant agreement No 777394, Project AIMS2-TRIALS). Additionally, the study was supported by the Spanish Ministry of Science and Innovation. Instituto de Salud Carlos III, co-financed by ERDF Funds from the European Commission, "A way of making Europe", CIBERSAM and Madrid Regional Government (B2017/BMD-3740 AGES-CM-2), European Union Structural Funds, Familia Alonso Foundation and Alicia Koplowitz Foundation.

\section{Availability of materials and data}

Research data are not shared. Codes and extended results can be obtained on reasonable request to corresponding author.

\section{References}


1. Hawkley, L. C. \& Cacioppo, J. T. Loneliness matters: A theoretical and empirical review of consequences and mechanisms. Ann. Behav. Med. 40, 218-227 (2010).

2. Qualter, P. et al. Loneliness Across the Life Span. Perspect. Psychol. Sci. 10, 250-264 (2015).

3. Spithoven, A. W. M., Bijttebier, P. \& Goossens, L. It is all in their mind: A review on information processing bias in lonely individuals. Clin. Psychol. Rev. 58, 97-114 (2017).

4. Cacioppo, J. T. \& Hawkley, L. C. Perceived social isolation and cognition. Trends in Cognitive Sciences vol. 13 447-454 (2009).

5. Vanhalst, J., Gibb, B. E. \& Prinstein, M. J. Lonely adolescents exhibit heightened sensitivity for facial cues of emotion. Cogn. Emot. 31, 377-383 (2017).

6. Bangee, M. \& Qualter, P. Examining the visual processing patterns of lonely adults. Scand. J. Psychol. 59, 351-359 (2018).

7. Cuthbert, B. N. \& Insel, T. R. Toward the future of psychiatric diagnosis: the seven pillars of RDoC. 18 (2013).

8. Mikami, A. Y., Miller, M. \& Lerner, M. D. Social functioning in youth with attention-deficit/hyperactivity disorder and autism spectrum disorder: transdiagnostic commonalities and differences. Clin. Psychol. Rev. 68, 54-70 (2019).

9. Schilbach, L. et al. Transdiagnostic commonalities and differences in resting state functional connectivity of the default mode network in schizophrenia and major depression. Neurolmage Clin. 10, 326-335 (2016).

10. Lara, E. et al. Does loneliness contribute to mild cognitive impairment and dementia? A systematic review and meta-analysis of longitudinal studies. Ageing Res. Rev. 52, 7-16 (2019).

11. Li, B. J. et al. Social isolation induces schizophrenia-like behavior potentially associated with HINT1, NMDA receptor 1, and dopamine receptor 2. Neuroreport 28, 462-469 (2017).

12. Kim, H. S. et al. Social cognition and neurocognition as predictors of conversion to psychosis in individuals at ultra-high risk. Schizophr. Res. 130, 170-175 (2011).

13. Sundström, A., Nordin Adolfsson, A., Nordin, M. \& Adolfsson, R. Loneliness increases the risk of allcause dementia and Alzheimer's disease. J. Gerontol. B. Psychol. Sci. Soc. Sci. XX, 1-8 (2019).

14. Galderisi, S., Mucci, A., Buchanan, R. W. \& Arango, C. Negative symptoms of schizophrenia: new developments and unanswered research questions. The Lancet Psychiatry 5, 664-677 (2018).

15. Badcock, J. C. et al. Loneliness in psychotic disorders and its association with cognitive function and symptom profile. Schizophr. Res. 169, 268-273 (2015).

16. Yang, C. et al. Different levels of facial expression recognition in patients with first- episode schizophrenia: A functional MRI study. 1-6 (2018) doi:10.1136/gpsych-2018-000014.

17. Maat, A., Haren, N. E. M. Van, Bartholomeusz, C. F., Kahn, R. S. \& Cahn, W. Emotion recognition and theory of mind are related to gray matter volume of the prefrontal cortex in schizophrenia. Eur. Neuropsychopharmacol. 26, 255-264 (2016). 
18. Fadel, B. T. M. D. M., De Carvalho, R. L. S., Dos Santos, T. T. B. A. \& Dourado, M. C. N. Facial expression recognition in Alzheimer's disease: A systematic review. J. Clin. Exp. Neuropsychol. 00, 112 (2018).

19. Park, S. et al. Behavioral and neuroimaging evidence for facial emotion recognition in elderly Korean adults with mild cognitive impairment, Alzheimer's disease, and frontotemporal dementia. Front. Aging Neurosci. 9, 1-17 (2017).

20. Bilderbeck, A. C. et al. Overview of the clinical implementation of a study exploring social withdrawal in patients with schizophrenia and Alzheimer's disease. Neurosci. Biobehav. Rev. 97, 87-93 (2019).

21. Kas, M. J. et al. A quantitative approach to neuropsychiatry: The why and the how. Neuroscience and Biobehavioral Reviews vol. 97 3-9 (2019).

22. American Psychiatric Association. Diagnostic and statistical manual of mental disorders (4th ed., text rev.). (Author, 2000).

23. Sheehan, D. V et al. The Mini-International Neuropsychiatric Interview (M.I.N.I): The development and validation of a structured diagnostic psychiatric interview for DSM-IV and ICD-10. J. Clin. Psychiatry 59, 22-33 (1998).

24. Jack, C. R. et al. NIA-AA Research Framework: Toward a biological definition of Alzheimer's disease. Alzheimer's and Dementia vol. 14 535-562 (2018).

25. Sheehan, D. V. et al. The Mini-International Neuropsychiatric Interview (M.I.N.I.): The development and validation of a structured diagnostic psychiatric interview for DSM-IV and ICD-10. J. Clin. Psychiatry 59, 22-33 (1998).

26. Birchwood, M., Smith, J., Cochrane, R., Wetton, S. \& Copestake, S. The Social Functioning Scale. The development and validation of a new scale of social adjustment for use in family intervention programmes with schizophrenic patients. Br. J. Psychiatry 157, 853-859 (1990).

27. De Jong-Gierveld, J. \& Kamphuls, F. The Development of a Rasch-Type Loneliness Scale. Appl. Psychol. Meas. 9, 289-299 (1985).

28. Rush, A. J. et al. The 16-Item Quick Inventory of Depressive Symptomatology. Biol. Psychiatry 54, 573-583 (2003).

29. Kay, S. R., Fiszbein, A. \& Opler, L. A. The positive and negative syndrome scale (PANSS) for schizophrenia. Schizophr. Bull. 13, 261-276 (1987).

30. Folstein, M. F., Folstein, S. E. \& McHugh, P. R. Mini-mental State. A practical method for grading the cognitive state of patiens for the clinician. J. Psychiatr. Res. 12, 189-198 (1975).

31. Lopez, M. N., Charter, R. A., Mostafavi, B., Nibut, L. P. \& Smith, W. E. Psychometric properties of the folstein Mini-Mental State Examination. Assessment 12, 137-144 (2005).

32. Jaeger, J. Digit symbol substitution test. The case for sensitivity over specificity in neuropsychological testing. J. Clin. Psychopharmacol. 38, 513-519 (2018).

33. Harmer, C. J. et al. Combined NK1 antagonism and serotonin reuptake inhibition: Effects on emotional processing in humans. J. Psychopharmacol. 27, 435-443 (2013). 
34. Montagne, B., Kessels, R. P. C., De Haan, E. H. F. \& Perrett, D. I. The emotion recognition task: A paradigm to measure the perception of facial emotional expressions at different intensities. Percept. Mot. Skills 104, 589-598 (2007).

35. Nakagawa, S., Johnson, P. C. D. \& Schielzeth, H. The coefficient of determination R2 and intra-class correlation coefficient from generalized linear mixed-effects models revisited and expanded. J. $R$. Soc. Interface 14, 20170213 (2017).

36. Freedman, M., Binns, M. A., Black, S. E., Murphy, C. \& Stuss, D. T. Theory of Mind and Recognition of Facial Emotion in Dementia. Alzheimer Dis. Assoc. Disord. 27, 56-61 (2013).

37. Bediou, B. et al. Impaired social cognition in mild Alzheimer disease. J. Geriatr. Psychiatry Neurol. (2009) doi:10.1177/0891988709332939.

38. Sapey-Triomphe, L. A. et al. Neuroanatomical correlates of recognizing face expressions in mild stages of Alzheimer's disease. PLoS One 10, 1-17 (2015).

39. Spilka, M. J., Arnold, A. E. \& Goghari, V. M. Functional activation abnormalities during facial emotion perception in schizophrenia patients and nonpsychotic relatives. Schizophr. Res. 168, 330-337 (2015).

40. Green, M. F., Horan, W. P. \& Lee, J. Social cognition in schizophrenia. Nat. Rev. Neurosci. 16, 620-631 (2015).

41. Haxby, J. V., Hoffman, E. A. \& Gobbini, M. I. Human neural systems for face recognition and social communication. Biol. Psychiatry 51, 59-67 (2002).

42. Cotter, J. et al. Social cognitive dysfunction as a clinical marker: A systematic review of metaanalyses across 30 clinical conditions. Neurosci. Biobehav. Rev. 84, 92-99 (2018).

43. Levine, J. Clinical convergence of autism, schizophrenia, and Alzheimer's disease: The case of social cognition. Neuroprotection in Autism, Schizophrenia and Alzheimer's Disease (Elsevier Inc., 2020). doi:10.1016/b978-0-12-814037-6.00002-1.

44. Kanai, R. et al. Brain structure links loneliness to social perception. Curr. Biol. 22, 1975-1979 (2012).

45. Dziura, S. L. \& Thompson, J. C. Social-Network Complexity in Humans Is Associated With the Neural Response to Social Information. Psychol. Scie 25, 2095-2101 (2014).

46. Lewis, P. A., Rezaie, R., Brown, R., Roberts, N. \& Dunbar, R. I. M. Ventromedial prefrontal volume predicts understanding of others and social network size. Neuroimage 57, 1624-1629 (2011).

47. Cacioppo, J. T., Norris, C. J., Decety, J., Monteleone, G. \& Nusbaum, H. In the eye of the beholder: Individual differences in perceived social isolation predict regional brain activation to social stimuli. J. Cogn. Neurosci. 21, 83-92 (2009).

\section{Tables}

Table 1. Descriptive statistics by study group. 


\begin{tabular}{|c|c|c|c|c|c|}
\hline & \multirow{2}{*}{\multicolumn{4}{|c|}{$\begin{array}{l}\text { Study } \\
\text { group }\end{array}$}} & \multirow{3}{*}{$\begin{array}{l}\text { Pairwise } \\
\text { differences }\end{array}$} \\
\hline & & & & & \\
\hline & SZc & SZ & $\mathrm{ADc}$ & $\mathrm{AD}$ & \\
\hline$n$ & 29 & 53 & 28 & 46 & \\
\hline Sex (men) & 58.62 & 71.70 & 53.57 & 55.56 & \\
\hline Age (years) & $\begin{array}{l}28.72 \\
(7.40)\end{array}$ & $\begin{array}{l}30.45 \\
(6.06)\end{array}$ & $\begin{array}{l}67.07 \\
(7.03)\end{array}$ & $\begin{array}{l}68.80 \\
(7.13)\end{array}$ & \\
\hline Race (white Caucasian) & 96.56 & 75.47 & 100.00 & 97.78 & \\
\hline Site (Spanish centres) & 48.26 & 39.62 & 25.00 & 42.22 & \\
\hline Education (years) & $\begin{array}{l}17.17 \\
(2.59)\end{array}$ & $\begin{array}{l}14.94 \\
(3.89)\end{array}$ & $\begin{array}{l}16.71 \\
(4.86)\end{array}$ & $\begin{array}{l}15.29 \\
(5.68)\end{array}$ & $\mathrm{SZc}>\mathrm{SZ}$ \\
\hline $\begin{array}{l}\text { Emotional disorder } \\
\text { comorbidity }\end{array}$ & 0 & 16.98 & 0 & 0 & $\mathrm{SZc}<\mathrm{SZ}$ \\
\hline Drug disorder comorbidity & 3.45 & 5.66 & 0 & 0 & \\
\hline General cognition & $\begin{array}{l}43.38 \\
(7.30)\end{array}$ & $\begin{array}{l}35.51 \\
(7.76)\end{array}$ & $\begin{array}{l}28.18 \\
(5.49)\end{array}$ & $\begin{array}{l}16.59 \\
(6.92)\end{array}$ & $\mathrm{SZc}>\mathrm{SZ} ; \mathrm{ADc}>\mathrm{AD}$ \\
\hline Depressive symptoms & $2.10(1.50)$ & $7.43(4.76)$ & $2.00(1.33)$ & $4.02(2.67)$ & $\mathrm{SZc}<\mathrm{SZ} ; \mathrm{ADc}<\mathrm{AD}$ \\
\hline \multicolumn{6}{|l|}{ Psychotic symptoms } \\
\hline Positive & & $\begin{array}{l}10.94 \\
(3.45)\end{array}$ & & & \\
\hline Negative & & $\begin{array}{l}14.26 \\
(6.08)\end{array}$ & & & \\
\hline Cognitive impairment & & & & $\begin{array}{l}23.98 \\
(1.99)\end{array}$ & \\
\hline
\end{tabular}

Note. Mean and standard errors (between brackets) are displayed for continuous variables. Percentage of participants are displayed for binary variables.

$\mathrm{SZ}=$ Schizophrenia group. $\mathrm{AD}=$ Alzheimer's disease group. $\mathrm{SZc}=\mathrm{SZ}$ healthy controls $. \mathrm{ADc}=\mathrm{AD}$ healthy controls.

Comorbidity with either emotional disorders (current diagnosis of depression episode, mania episode, hypomania episode; type I or II bipolar disorder; panic disorder, agoraphobia, social phobia, generalised anxiety disorder) or drug disorders (current diagnosis of alcohol dependence, alcohol abuse disorder, other drug dependence or other drug abuse disorder) were assessed using the International Neuropsychiatric Interview (MINI).

General cognition was assessed by means of the digit symbol substitution test (total score = correct responses incorrect responses). Depressive symptoms were measured using the Quick Inventory of Depressive Symptomatology (QIDS-SR16). Psychotic symptoms were assessed using the Positive and Negative Syndrome Scale (PANSS). Cognitive impairment was measured using the Mini-Mental State Examination (MMSE).

Pairwise comparison tests were conducted using the Student's $t$ test (for continuous outcomes) or $\mathrm{c}^{2}$-based test (for binary outcomes), under the Bonferroni's correction ( $p$-value cut-off $=.008)$. 
Table 2. FERT outcomes by study group.

\begin{tabular}{|c|c|c|c|c|c|c|c|}
\hline & \multicolumn{4}{|l|}{$\begin{array}{l}\text { Study } \\
\text { group }\end{array}$} & \multirow[t]{2}{*}{$F$ statistic } & \multirow[t]{2}{*}{$\begin{array}{l}\text { Effect } \\
\text { size }\end{array}$} & \multirow[t]{2}{*}{ Pairwise differences } \\
\hline & SZc & $\mathrm{SZ}$ & $\mathrm{ADc}$ & $\mathrm{AD}$ & & & \\
\hline Positive emotions & & & & & $11.51 * *$ & 0.01 & \\
\hline Accuracy & $\begin{array}{l}59.10 \\
(6.12)\end{array}$ & $\begin{array}{l}54.83 \\
(10.21)\end{array}$ & $\begin{array}{l}53.77 \\
(8.59)\end{array}$ & $\begin{array}{l}47.13 \\
(10.78)\end{array}$ & $13.12^{* *}$ & 0.07 & $\begin{array}{l}\mathrm{AD}<\text { all other groups; } \mathrm{SZ} \\
<\mathrm{SZC}\end{array}$ \\
\hline Misclassification & $\begin{array}{l}3.16 \\
(3.15)\end{array}$ & $\begin{array}{l}6.71 \\
(7.31)\end{array}$ & $\begin{array}{l}6.40 \\
(4.73)\end{array}$ & $\begin{array}{l}13.97 \\
(10.98)\end{array}$ & $17.82 * *$ & 0.11 & $\mathrm{AD}>$ all other groups \\
\hline Negative emotions & & & & & $20.51 * *$ & 0.15 & \\
\hline Accuracy & $\begin{array}{l}65.02 \\
(5.73)\end{array}$ & $\begin{array}{l}55.35 \\
(13.95)\end{array}$ & $\begin{array}{l}51.37 \\
(9.09)\end{array}$ & $\begin{array}{l}35.04 \\
(14.04)\end{array}$ & $51.59 * *$ & 0.22 & $\begin{array}{l}\text { SZc > all other groups; AD } \\
<\text { all other groups }\end{array}$ \\
\hline Misclassification & $\begin{array}{l}7.14 \\
(4.53)\end{array}$ & $\begin{array}{l}9.80 \\
(8.12)\end{array}$ & $\begin{array}{l}8.73 \\
(5.20)\end{array}$ & $\begin{array}{l}9.76 \\
(8.54) \\
\end{array}$ & 1.65 & 0.03 & \\
\hline
\end{tabular}

Note. Mean and standard errors (between brackets) are displayed.

$\mathrm{SZ}=$ Schizophrenia group. $\mathrm{AD}=$ Alzheimer's disease group. $\mathrm{SZc}=\mathrm{SZ}$ healthy controls. $\mathrm{ADc}=\mathrm{AD}$ healthy controls.

The Snedecor's F-based statistic was derived from multivariate analysis of covariance (for both positive and negative emotion outcomes) and univariate analysis of covariance (for accuracy and misclassification rate scores). In both cases, the study group (controls vs. schizophrenia group vs. Alzheimer's disease group) was used as a between-group factor and the age group (younger vs. older participants) as a covariate. The $F$ statistic and the $\mathrm{h}^{2}$ partial statistic (effect size estimate) were derived from the group effect.

Pairwise comparison tests were conducted using the Student's $t$ test, comparing the four study groups under the Bonferroni's correction ( $p$-value cut-off $=.008)$.

$* p<.05 ;{ }^{* *} p<.01$.

Table 3. Regression coefficients of covariates for FERT outcomes. 


\begin{tabular}{|c|c|c|c|c|c|c|c|c|c|}
\hline & \multicolumn{6}{|c|}{ Positive emotion recognition } & \multirow{2}{*}{\multicolumn{3}{|c|}{$\begin{array}{l}\text { Negative emotion } \\
\text { recognition } \\
\text { Accuracy }\end{array}$}} \\
\hline & Accuracy & & & Misc & fication & & & & \\
\hline Predictor & $B$ & $\mathrm{CI}_{95}$ & $t$ & $B$ & $C_{95}$ & $t$ & $B$ & $\mathrm{CI}_{95}$ & $t$ \\
\hline Intercept & 57.71 & $\begin{array}{l}57.50 \\
58.00\end{array}$ & $3.42^{* *}$ & 7.57 & $\begin{array}{l}7.16 \\
10.39\end{array}$ & 1.33 & 52.75 & $\begin{array}{l}51.61 \\
52.85\end{array}$ & $5.68^{* *}$ \\
\hline Sex (ref.: man) & 3.89 & $\begin{array}{l}3.73 \\
4.01\end{array}$ & $2.52 *$ & -3.48 & $\begin{array}{l}-3.51 \\
-3.37\end{array}$ & $-2.83 * *$ & 4.73 & $\begin{array}{l}4.73 \\
4.88\end{array}$ & $2.47^{*}$ \\
\hline Age & -0.17 & $\begin{array}{l}-0.18 \\
-0.17\end{array}$ & $-3.03 *$ & 0.04 & $\begin{array}{l}0.02 \\
0.04\end{array}$ & 0.57 & -0.15 & $\begin{array}{l}-0.16 \\
-0.15\end{array}$ & -1.26 \\
\hline General cognition & & & & -0.25 & $\begin{array}{l}-0.28 \\
-0.25\end{array}$ & $-2.85 * *$ & 0.44 & $\begin{array}{l}0.43 \\
0.48\end{array}$ & $3.19 * *$ \\
\hline $\begin{array}{l}\text { Depressive } \\
\text { symptoms }\end{array}$ & & & & 0.05 & $\begin{array}{l}0.05 \\
0.10\end{array}$ & 0.2 & -0.12 & $\begin{array}{l}-0.13 \\
-0.11\end{array}$ & -0.31 \\
\hline $\begin{array}{l}\text { Emotional } \\
\text { comorbidity }\end{array}$ & & & & -0.27 & $\begin{array}{l}-0.29 \\
0.17\end{array}$ & -0.08 & 4.8 & $\begin{array}{l}4.78 \\
5.12\end{array}$ & 0.91 \\
\hline Social engagement & & & & 0.16 & $\begin{array}{l}0.15 \\
0.17\end{array}$ & $2.48 *$ & -0.31 & $\begin{array}{l}-0.31 \\
-0.30\end{array}$ & $-3.12 * *$ \\
\hline $\begin{array}{l}\text { Interpersonal } \\
\text { behaviour }\end{array}$ & & & & -0.01 & $\begin{array}{l}-0.01 \\
-0.01\end{array}$ & -0.22 & 0.06 & $\begin{array}{l}0.06 \\
0.07\end{array}$ & 0.95 \\
\hline Loneliness & & & & 0.02 & $\begin{array}{l}0.01 \\
0.05\end{array}$ & 0.06 & -0.18 & $\begin{array}{l}-0.19 \\
-0.16\end{array}$ & -0.41 \\
\hline Random-effects $S D$ & 2.04 & & & 2.72 & & & 5.93 & & \\
\hline Conditional $R^{2}$ & 0.07 & & & 0.15 & & & 0.24 & & \\
\hline \multicolumn{10}{|l|}{ AIC } \\
\hline Unconstrained & 1172.02 & & & 1112 & & & 1255.84 & & \\
\hline Sociodeographic & 1166.04 & & & 1107 & & & 1250.08 & & \\
\hline Full model & 1173.49 & & & 1104 & & & 1235.81 & & \\
\hline
\end{tabular}

Note. Outcomes were the facial emotion recognition task (FERT) measures. Parameters are shown for the models with better fit. Covariates were modelled under a mixed-effects solution, including the study group as a multilevel factor and the recruitment site as a weighting factor. All the covariates were centred (except sex and emotional disorder comorbidity) to make intercept easier to be understood.

General cognition was assessed by means of the digit symbol substitution test. Depressive symptoms were measured using the Quick Inventory of Depressive Symptomatology (QIDS-SR16). Social engagement and Interpersonal behaviour scores were taken from the related Social Functioning Scale factors. The Loneliness and Affiliation Scale was used to obtain the loneliness score.

Comorbidity with emotional disorders involved having diagnosed an emotional disorder (current diagnosis of depression episode, mania episode, hypomania episode; type I or II bipolar disorder; panic disorder, agoraphobia, social phobia, generalised anxiety disorder) in base of the International Neuropsychiatric Interview (MINI).

Unconstrained model $=$ Regression model without covariates. Covariates model $=$ Regression model with sociodemographic covariates (the significant model for the positive emotion accuracy). Full model $=$ Regression 
model with all the covariates (the significant model for the positive emotion misclassification and the negative emotion accuracy).

$B=$ Regression loading. $C I_{95}=95 \%$ confidence interval of the $B . t=$ Student's $t$ statistic. $S D=$ Standard deviation. Conditional $R^{2}=$ Nakagawa's coefficient of determination $R^{2}$. AIC = Akaike information criterion.

$* p<.05 ; * * p<.01$.

\section{Figures}

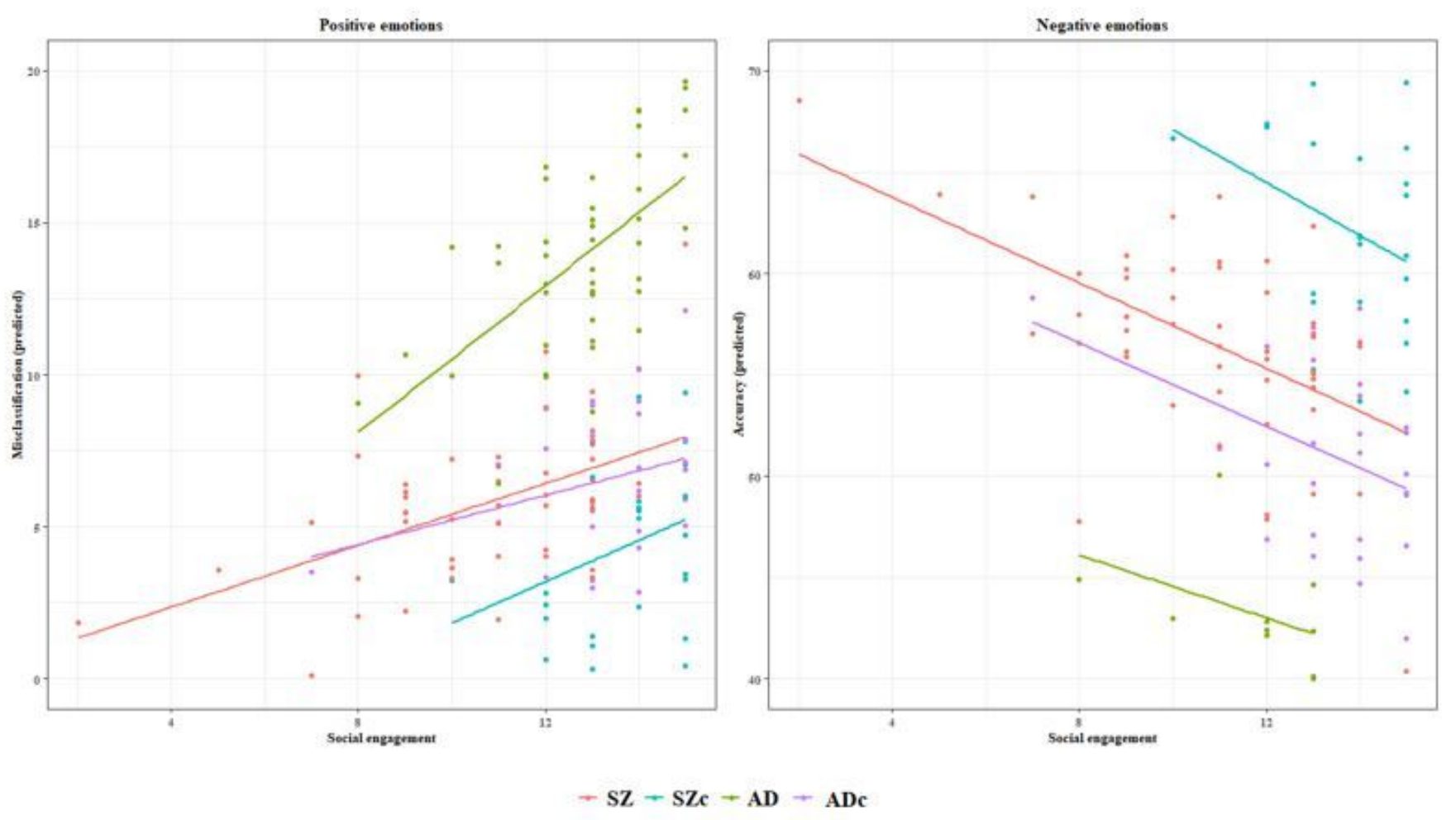

Figure 1

Predicted accuracy and misclassification in emotion recognition according to social engagement and study groups. Note. Regression lines linking the misclassification rate for both positive emotions (happiness and surprise) and the accuracy rate for negative emotions (sadness, fear, disgust, anger), and social engagement. Turquoise line $=$ Schizophrenia healthy controls (SZc). Red line $=$ Schizophrenia group (SZ). Violet line = Alzheimer's disease healthy controls (ADC). Green line = Alzheimer's disease group (AD). 